\title{
Changes in resting state functional connectivity after repetitive transcranial direct current stimulation applied to motor cortex in fibromyalgia patients
}

Chelsea M. Cummiford ${ }^{1,2^{*}+}$, Thiago D. Nascimento ${ }^{3,4+}$, Bradley R. Foerster ${ }^{5,6,7}$, Daniel J. Clauw ${ }^{2,8}$, Jon-Kar Zubieta ${ }^{9}$, Richard E. Harris ${ }^{1,2,8+}$ and Alexandre F. DaSilva ${ }^{3,4+}$

\begin{abstract}
Background: Fibromyalgia (FM) is a chronic, centralized pain condition characterized by alterations in the functional, chemical, and structural brain networks responsible for sensory and mood processing. Transcranial direct current stimulation (tDCS) has emerged as a potential treatment for FM. tDCS can alter functional connectivity (FC) in brain regions underneath and distant to the stimulating electrode, although the analgesic mechanisms of repetitive tDCS remain unknown. The aim of this study was to investigate how a clinically relevant schedule of tDCS sessions alters resting state FC and how these changes might relate to clinical pain.

Methods: Resting state functional magnetic resonance imaging data were collected from 12 patients with FM at baseline, after 5 days of sham treatment, and after 5 days of real tDCS with the anode over the left primary motor cortex (M1) and the cathode over the right supraorbital cortex. Seed to whole-brain FC analyses were performed with seed regions placed in bilateral M1, primary somatosensory cortices (S1), ventral lateral $(\mathrm{VL})$ and ventral posterolateral (VPL) thalami, and periaqueductal gray (PAG).

Results: Stronger baseline FC between M1-VL thalamus, S1-anterior insula, and VL thalamus-PAG predicted greater analgesia after sham and real tDCS. Sham treatment (compared with baseline) reduced FC between the VPL thalamus, S1, and the amygdala. Real tDCS (compared with sham treatment) reduced FC between the VL thalamus, medial prefrontal, and supplementary motor cortices. Interestingly, decreased FC between the VLNPL thalamus and posterior insula, M1, and S1 correlated with reductions in clinical pain after both sham and active treatments.

Conclusions: These results suggest that while there may be a placebo response common to both sham and real tDCS, repetitive $\mathrm{M} 1 \mathrm{tDCS}$ causes distinct changes in FC that last beyond the stimulation period and may produce analgesia by altering thalamic connectivity.
\end{abstract}

Keywords: Repetitive transcranial direct current stimulation (tDCS), Fibromyalgia, Functional connectivity, Resting state, Motor cortex, fMRI

\footnotetext{
*Correspondence: chelsmar@umich.edu

'Equal contributors

${ }^{1}$ Neuroscience Graduate Program, University of Michigan, Ann Arbor, MI, USA

${ }^{2}$ Chronic Pain and Fatigue Research Center, Department of Anesthesiology,

University of Michigan, Ann Arbor, MI, USA

Full list of author information is available at the end of the article
} 


\section{Background}

Fibromyalgia (FM) is a chronic centralized pain condition characterized by widespread pain, fatigue, sleep problems, cognitive dysfunction, and mood disturbances [1]. While the exact pathophysiology of FM remains unknown, a prevailing hypothesis states that a sensory processing dysfunction within the central nervous system creates, amplifies, or sustains the perception of chronic pain [2]. In support of this hypothesis, brain network alterations seen in these patients fall into two broad categories: decreased descending antinociceptive transmission, and/or enhanced pronociceptive processing [3-6].

Motor cortical dysfunction has been suggested in a number of chronic pain conditions, including FM. In general, the primary motor cortex (M1) shows increased cortical excitability at baseline and heightened responses to sensory stimuli, which may be suggestive of a reduction in inhibitory activity [7]. Noninvasive brain stimulation has emerged as an attractive therapeutic option for chronic pain conditions, given its ability to target specific cortical regions. Researchers in some studies have reported that transcranial direct current stimulation (tDCS) over M1 relieves pain in FM [8-10]. However, the authors of a recent review did not find a significant difference between sham and real M1 tDCS on shortterm pain relief [11]. The lack of effect may be due to significant heterogeneity between the studies (i.e., stimulation parameters, number of treatment sessions, type of chronic pain) included in the review. It is also possible that sham $\mathrm{tDCS}$ produces a significant placebo response. Consistent with previous work implicating the endogenous opioid system in placebo analgesia [12, 13], we recently showed that sham $\mathrm{tDCS}$ caused the release of endogenous opioids in the periaqueductal gray (PAG), precuneus, and thalamus [14].

While placebo responses are clearly present in tDCS, the specific neurobiology underlying the analgesic effects of real tDCS are less clear. During and immediately after stimulation, tDCS may alter excitability by modulating resting membrane potential. Longer-lasting effects may be due to changes in synaptic plasticity via mechanisms similar to long-term potentiation or depression [15]. M1 tDCS can alter the functional connectivity (FC) of regions under the stimulating electrode [16], as well as spatially distant but structurally connected regions, such as the thalamus [17] and dorsolateral prefrontal cortex $[18,19]$. Real tDCS also acts on the endogenous opioid system [14] similarly to invasive motor cortex stimulation (MCS) [20, 21]. However, these studies were conducted in healthy participants and the investigators examined FC during or shortly after M1 tDCS. There have been no investigations of how M1 tDCS alters resting state $\mathrm{FC}$ in patients with chronic pain treated repeatedly, as they might be in clinical practice.
We measured clinical pain and resting state FC in 12 patients with FM at baseline, after 5 days of sham treatment, and after 5 days of real tDCS. We were interested in three questions: (1) Does baseline connectivity predict clinical treatment response? (2) Are there differences in FC after sham and real tDCS? (3) Do changes in FC relate to analgesia? We hypothesized that strong M1-thalamus connectivity at baseline would predict a better clinical response, as shown in previous M1 stimulation studies $[22,23]$. In addition, because we found a trend toward decreased glutamate + glutamine (Glx) in the thalamus after real tDCS in these same patients [24], and given the strong structural connectivity between M1 and the thalamus [25], we hypothesized that real tDCS would decrease FC between the thalamus and brain regions involved in pain perception.

\section{Methods}

\section{Patients}

We recruited 13 female patients with FM (age range 27-64 years, mean \pm standard deviation $47.6 \pm 10.6$ years) for this study. One patient dropped out after the baseline visit, and the remaining twelve patients completed the entire protocol. All patients met the 1990 American College of Rheumatology criteria for FM [1], had experienced symptoms for at least 1 year, and reported pain on more than $50 \%$ of days. The inclusion criteria were righthanded, a body mass index of 36 or less, and agreement to delay taking new medications or treatments for FM during the study. The exclusion criteria were pregnant or breastfeeding, participation in other clinical trials, currently taking opiates, history of autoimmune or chronic inflammatory disease that causes pain, substance abuse or severe psychiatric illness, and contraindications for magnetic resonance imaging procedures. The University of Michigan Institutional Review Board approved this study, and all subjects gave written informed consent. The effect of tDCS on brain metabolites in these same subjects is described in a previous report [24].

\section{Study design}

Our within-subjects crossover design had three phases (Additional file 1: Figure S1). Session 1 included a baseline pain assessment and functional magnetic resonance imaging (fMRI). Session 2 consisted of sham tDCS for 5 consecutive days followed by pain assessment and a second fMRI. Session 3 comprised real tDCS for 5 consecutive days followed by pain assessment and a third fMRI 3. The sham and real tDCS phases were separated by a 7 - to 11-day washout period (mean 9.9 days). We chose to perform real tDCS for 5 consecutive days because previous studies in patients with FM have shown a meaningful reduction in clinical pain using this protocol $[8,9]$. To limit carryover from real to sham tDCS, we 
did not use a randomized design [26]. All participants were debriefed during a final follow-up visit. Patients were also offered a clinical referral to an outpatient clinic at our institution for continuation of care with regular therapy for their symptoms.

\section{Clinical pain outcomes}

Clinical pain intensity was assessed as an "average" experience for the week before each assessment using a visual analogue scale (VAS), with 0 being "no pain" and 10 being "worst possible pain." Clinical pain was also assessed using the short-form McGill Pain Questionnaire [27], and affective state was measured using the Positive and Negative Affect Schedule (PANAS) [28]. We were missing McGill baseline pain data for one patient, PANAS scores across all conditions for one patient, and PANAS baseline-only scores for two patients. The clinical results have been published previously [24] and are provided in Additional file 1: Table S1. Differences in clinical variables across conditions were assessed with repeated-measures analysis of variance (ANOVA) using IBM SPSS version 22 software (IBM, Armonk, NY, USA). Significance was set at an $\alpha$ level of $p<0.05$. The changes in clinical pain scores used in neuroimaging analyses were calculated by subtraction of sham - baseline VAS and real - sham VAS.

\section{tDCS protocol}

The tDCS protocol was carried out as described previously [29]. Briefly, for both sham and real tDCS sessions, the anode electrode was placed on the scalp over the left motor cortex and the cathode was positioned over the right supraorbital cortex. Positions were determined individually using the 10-20 international system of electroencephalogram electrode placement at C3 and FP2, respectively. Electrodes were placed by the same operators (AFD and TDN) for all patients. Active stimulation consisted of $2 \mathrm{~mA}$ of current applied continuously for 20 minutes. During sham tDCS, the current was applied for 30 seconds at the beginning and end of the session. Patients were blinded to type of treatment (i.e., real vs. sham) they were receiving. This protocol is identical to that used in previous studies of M1 tDCS in patients with FM $[8,9]$.

\section{Neuroimaging methods}

Resting state fMRI sessions were performed on an Ingenia 3.0 T system (Philips Medical Systems, Best, the Netherlands) with a 15-channel receive head coil. Each scan lasted 10 minutes, and parameters included a T2*weighted blood oxygen level-dependent (BOLD) echoplanar imaging sequence [repetition time (TR) $2000 \mathrm{ms,}$ echo time (TE) $30 \mathrm{~ms}$, flip angle 77 degrees, 30 slices, voxel size $3.44 \times 3.33 \times 4.00 \mathrm{~mm}$ ]. Physiological data (cardiac and respiratory volume) were collected simultaneously. A high-resolution structural image was acquired for normalization purposes (TR/TE 9.8/4.6 ms, flip angle 8 degrees, 151 slices, voxel size $1 \times 1 \times 1 \mathrm{~mm}$ ). fMRI data were checked for quality and head motion greater than $3 \mathrm{~mm}$; no data were excluded. Resting state fMRI data were preprocessed using SPM8 (http://www. fil.ion.ucl.ac.uk/spm/software/spm8/) running on MATLAB R2010a (MathWorks, Natick, MA, USA) and included physiological artifact correction, slice timing correction, realignment, coregistration, normalization to Montreal Neurological Institute space, and smoothing (full width at half maximum $8 \mathrm{~mm}$ ).

Seed to whole-brain FC analyses were performed using the Conn Toolbox [30]. Seeds were chosen on the basis of the following criteria: (1) location under stimulating anode (left precentral gyrus and left postcentral gyrus [M1/primary somatosensory cortex (S1); WFU PickAtlas (http://www.nitrc.org/projects/wfu_pickatlas)], (2) structural connectivity to left M1 and S1 [right pre- and postcentral gyri, bilateral ventral lateral (VL) and ventral posterolateral (VPL) thalamus; WFU PickAtlas), and (3) our previous tDCS studies (PAG [14]). The time series for each seed region was extracted and white matter, cerebrospinal fluid signal, and realignment parameters were entered into the analysis as regressors of no interest. A band-pass filter $(0.008-0.09 \mathrm{~Hz})$ was applied to remove linear drift artifacts and high-frequency noise. First-level analyses were performed by correlating the time series from each seed region with the rest of the voxels in the brain, creating seed to whole-brain Fishertransformed correlation maps. These maps were imported into SPM8 for group-level analyses.

For prediction analyses, we performed seed to wholebrain regression analyses with baseline FC maps and change in clinical pain (real - baseline) as a regressor of interest. Main effects were calculated using repeatedmeasures ANOVA design with baseline, sham, and real tDCS FC maps. The contrasts of interest were baseline versus sham $t D C S$ and sham versus real $t D C S$. We also examined the change in FC across the entire study using the contrast baseline versus real tDCS. To examine correlations between changes in connectivity and changes in clinical pain, we first created difference images by subtracting first-level connectivity maps for each subject (sham - baseline, real-sham, and real-baseline). We then performed a regression analysis with VAS change scores as a regressor of interest. All analyses were controlled for differences in age. Results were thresholded at uncorrected $p<0.001$ on the voxel level and $p<0.05$ familywise error (FWE) correction for multiple comparisons at the cluster level with a cluster size of greater than 5 voxels. For a priori regions that did not meet this stringent threshold, we performed small volume corrections 
(SVCs) using the anatomically (WFU PickAtlas) defined regions of interest (ROIs) used as seed regions or functionally defined ROIs derived from our previous findings in FM [6]. Significance for SVC was set at $p<0.05$ FWE at the cluster level with a cluster size of greater than 5 voxels. The Fisher-transformed correlation values were extracted using MarsBaR software (http://marsbar.sourceforge.net/) and post hoc analyses performed using IBM SPSS version 22 software.

\section{Results}

\section{Clinical pain reduction with sham and real tDCS}

As reported previously [24], there was a trend toward improvement in VAS clinical pain during the sham period [mean difference \pm standard error (SE) for sham minus baseline $-1.042 \pm 0.572$, $95 \%$ confidence interval (CI) -0.218 to $2.301 ; p=0.096$, and there was no significant difference in pain relief between sham and real tDCS (mean difference $\pm \mathrm{SE}$ for real minus sham $-0.750 \pm 0.494$, $95 \% \mathrm{CI}-1.838$ to $0.338 ; p=0.157$ ). However, clinical pain significantly decreased across the entire study from baseline to after real tDCS (mean difference \pm SE for real minus baseline $-1.792 \pm 0.762,95 \% \mathrm{CI}-3.470$ to -0.114 ; $p=0.038$ ). There were no significant differences in clinical pain as measured by the McGill Pain questionnaire or PANAS positive affect. There was a significant difference between baseline and real tDCS in PANAS negative affect (mean difference $\pm \mathrm{SE}$ for real minus baseline $-3.0 \pm 1.067$, $95 \%$ CI -5.461 to $-0.539 ; p=0.023)$. Clinical results for each patient individually across the study are presented in Additional file 1: Table S2.

\section{Stronger baseline FC is associated with subsequent analgesia}

To examine the common predictive ability of baseline FC for reductions in pain across sham and real tDCS (as this was where the significant clinical effect on pain was found), we used predefined ROIs and correlated baseline FC with improvements in clinical pain across the entire study period (real - baseline). Patients who had stronger connectivity at baseline between the left M1 seed and left VL thalamus $(p=0.011$ FWE, SVC), between the left
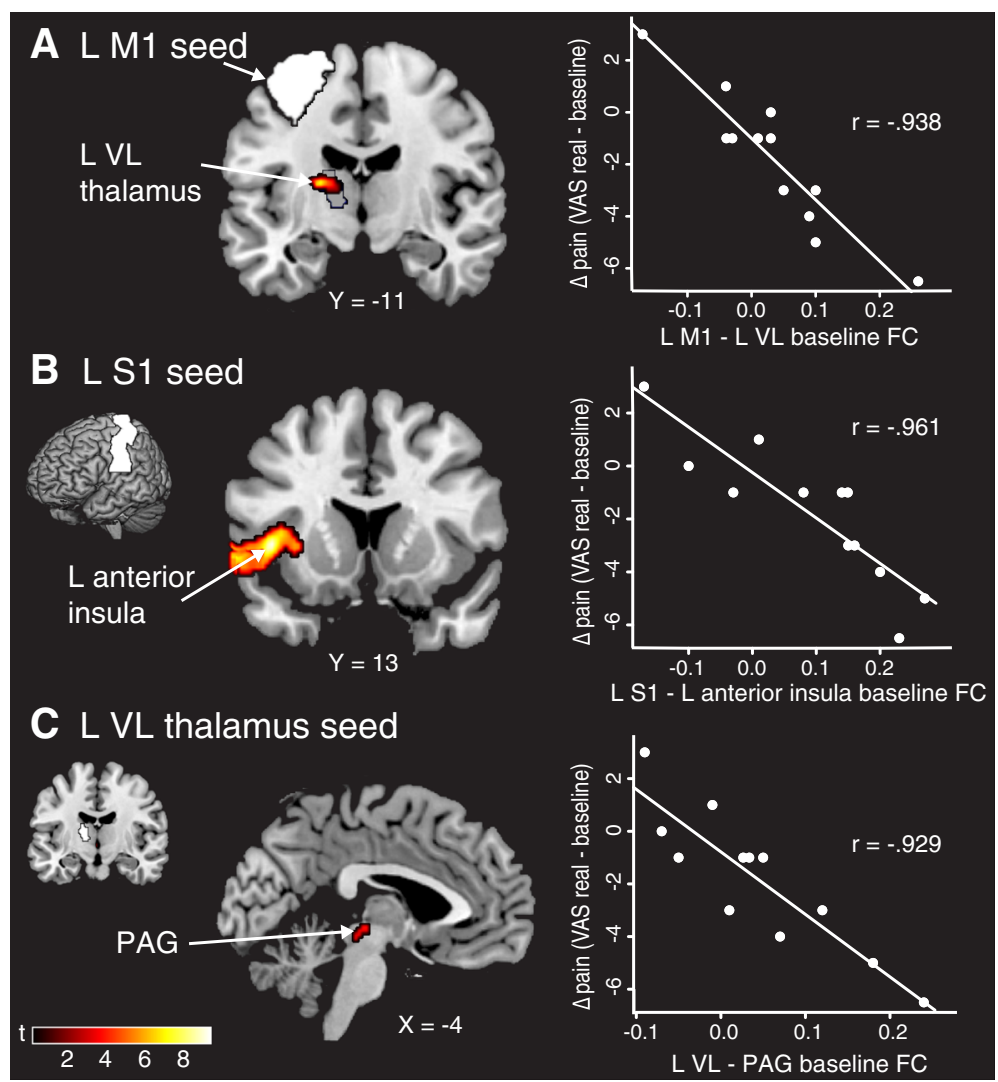

Fig. 1 Stronger FC at baseline predicts analgesia. a Patients with higher L M1 (seed in white) - L VL (anatomical region outlined in black) connectivity at baseline had a greater reduction in clinical pain across sham and real transcranial direct current stimulation (tDCS) periods (displayed at $p=0.005$ ). b Stronger L S1 (seed in white) - L anterior insula FC at baseline predicted a better clinical response. c Connectivity between the $L$ VL thalamus (seed in white) and the PAG at baseline also predicted patients who would respond to sham and real tDCS treatment. Data shown are Fisher-transformed $r$ values. M1 primary motor cortex, VL ventral lateral, S1 primary somatosensory cortex, PAG periaqueductal gray, VAS visual analogue scale, $L$ left, $R$ right, FC functional connectivity 
S1 seed and left anterior insula ( $p=0.001 \mathrm{FWE})$, and between the left VL thalamus seed and PAG $(p=0.007$ FWE, SVC) had greater improvement in clinical pain scores across sham and real tDCS periods (Fig. 1 and Table 1). Importantly, these correlations were also significant when we looked at change in clinical pain from baseline to sham or from sham to real tDCS alone (with one exception: left VL-PAG baseline FC and real-sham clinical pain; $p=0.057$ ) (Additional file 1: Table S3). There were no regions that showed significant correlations between less connectivity at baseline and better treatment response (Additional file 1: Figure S2). In post hoc analyses, there were no significant correlations between baseline connectivity of these regions and the change McGill clinical pain or the change in positive and negative affect.

\section{Sham tDCS is associated with decreases in FC}

Because previous studies have shown a placebo analgesic response on experimental and clinical pain during sham tDCS [31], we examined whether sham tDCS changed resting state FC (sham-baseline) (Table 2). After five sessions of sham tDCS, patients with FM had reduced FC between the left VPL thalamus seed and left S1 ( $p=0.016 \mathrm{FWE})$, left amygdala/parahippocampal gyrus $(p=0.004 \mathrm{FWE})$, and right inferior parietal lobule (IPL) $(p=0.013$ FWE) (Fig. 2a). FC also decreased between the right VPL thalamus seed and left IPL $(p=0.049$ FWE) (Fig. 2b), between the PAG seed and precuneus $(p=0.001$ FWE) (Fig. 2c), and between the right M1 seed and right cerebellum $(p=0.002 \mathrm{FWE})$. There were no significant increases in FC after sham compared with baseline (Additional file 1: Figure S3).

To determine if changes in FC were related to changes in clinical pain during the sham period, we ran a regression analysis with each participant's connectivity difference map (sham - baseline) with change in VAS (sham - baseline) as a regressor of interest (Table 3). The change in connectivity

Table 1 Predicting changes in clinical pain from baseline functional connectivity

\begin{tabular}{|c|c|c|c|c|c|c|c|}
\hline $\begin{array}{l}\text { Seed } \\
\text { FC region }\end{array}$ & \multicolumn{3}{|c|}{$\begin{array}{l}\text { MNI coordinates } \\
(x, y, z)\end{array}$} & \multirow[t]{2}{*}{$r$ Value } & \multirow[t]{2}{*}{$\mathrm{T}$} & \multirow[t]{2}{*}{$\begin{array}{l}\text { Cluster } \\
\text { size }\end{array}$} & \multirow[t]{2}{*}{$\begin{array}{l}\text { Cluster } \\
p \text { value }\end{array}$} \\
\hline LM1 & & & & & & & \\
\hline L VL thalamus & -18 & -14 & 12 & -0.938 & 5.41 & 7 & 0.011 FWE $^{*}$ \\
\hline \multicolumn{8}{|l|}{ L S1 } \\
\hline $\begin{array}{l}\mathrm{L} \text { anterior } \\
\text { insula }\end{array}$ & -42 & 14 & 2 & -0.961 & 9.38 & 396 & 0.001 FWE \\
\hline \multicolumn{8}{|l|}{ L VL thalamus } \\
\hline PAG & -6 & -26 & -8 & -0.929 & 5.25 & 8 & 0.007 FWE* \\
\hline
\end{tabular}

FC functional connectivity, $L$ left, $M 1$ primary motor cortex, $V L$ ventral lateral, S1 primary somatosensory cortex, PAG periaqueductal gray, MNI Montreal Neurological Institute $T$ test statistic

*Significant at $p<0.05$ with small volume correction
Table 2 Main effect of sham tDCS on FC

\begin{tabular}{|c|c|c|c|c|c|c|}
\hline \multirow{2}{*}{$\begin{array}{l}\text { Seed } \\
\text { FC region } \\
\text { Baseline > sham }\end{array}$} & \multicolumn{3}{|c|}{$\begin{array}{l}\text { MNI coordinates } \\
(x, y, z)\end{array}$} & \multirow[t]{2}{*}{$\mathrm{T}$} & \multirow[t]{2}{*}{$\begin{array}{l}\text { Cluster } \\
\text { size }\end{array}$} & \multirow[t]{2}{*}{$\begin{array}{l}\text { Cluster } \\
p \text { value }\end{array}$} \\
\hline & & & & & & \\
\hline \multicolumn{7}{|l|}{ L VPL } \\
\hline LS1 & -62 & -16 & 42 & 6.80 & 304 & 0.016 FWE \\
\hline L parahipp/amyg & -32 & -14 & -26 & 6.70 & 408 & $0.004 \mathrm{FWE}$ \\
\hline $\mathrm{R} I P L$ & 44 & -36 & 32 & 5.69 & 320 & 0.013 FWE \\
\hline \multicolumn{7}{|l|}{ R VPL } \\
\hline LIPL & -34 & -36 & 34 & 5.28 & 230 & $0.049 \mathrm{FWE}$ \\
\hline \multicolumn{7}{|c|}{ R M1 (precentral gyrus) } \\
\hline R cerebellum & 16 & -74 & -36 & 5.91 & 485 & $0.002 \mathrm{FWE}$ \\
\hline \multicolumn{7}{|l|}{ PAG } \\
\hline Precuneus & -20 & -84 & 24 & 4.88 & 618 & $0.001 \mathrm{FWE}$ \\
\hline \multicolumn{7}{|l|}{ Baseline < sham } \\
\hline N.S. & & & & & & \\
\hline
\end{tabular}

between the left VL thalamus seed and the left posterior insula was positively correlated with change in clinical pain $(p=0.001 \mathrm{FWE})$. Patients with reduced connectivity between the left VL thalamus and posterior insula had a greater reduction in pain intensity after sham tDCS (Fig. 3a). Reduced connectivity between the right VPL thalamus seed and right M1 $(p=0.001 \mathrm{FWE})$, right S1 $(p=0.008 \mathrm{FWE})$, and left M1 $(p=0.046 \mathrm{FWE})$ also correlated with reduced pain after sham tDCS (Fig. 3b). Decreased FC between the right $\mathrm{S} 1$ seed and the cerebellum ( $p=0.001 \mathrm{FWE})$ was also positively correlated with change in pain. These changes in connectivity were not significantly correlated with changes in positive and negative affect or McGill clinical pain. There were no significant relationships between increases in connectivity and decreases in clinical pain (Additional file 1: Figure S6).

\section{Real tDCS is also associated with decreases in FC}

Next, we measured changes in FC between sham and real tDCS (Table 4). After real tDCS, FC decreased between the left VL thalamus seed and the medial prefrontal cortex $(\mathrm{mPFC})(p=0.006 \mathrm{FWE})$ and left supplementary motor area (SMA) ( $p=0.043$ FWE) (Fig. 4a). FC also decreased between the right VL thalamus seed and the cerebellum $(p=0.001 \mathrm{FWE})$ and left SMA ( $p=0.016$ FWE) (Fig. 4b). There were no significant increases in FC (Additional file 1: Figure S3).

When we compared baseline with real tDCS, we found significant decreases in connectivity between the left VPL thalamus seed and the left IPL $(p=0.041$ FWE) and between the PAG seed and the posterior cingulate $(p=0.007$ 


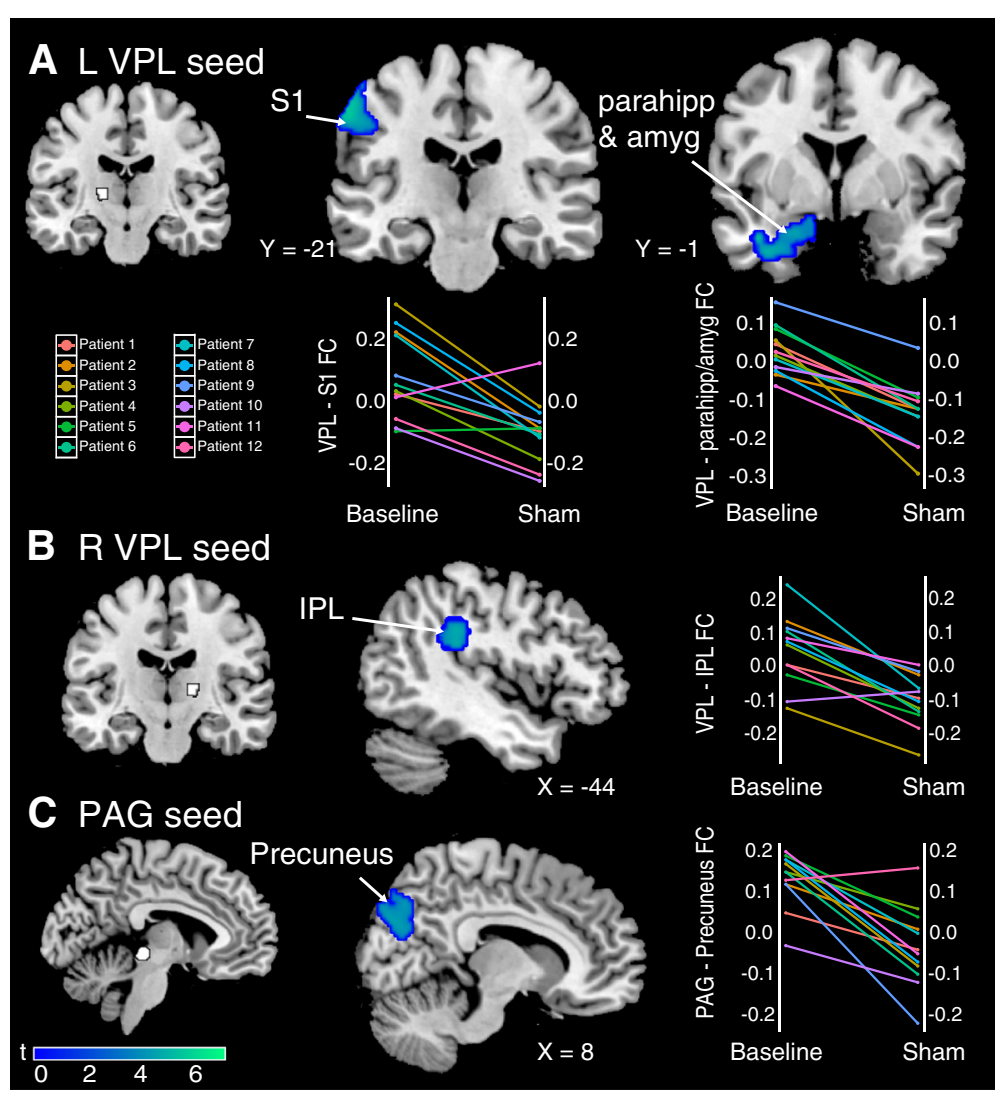

Fig. 2 Sham transcranial direct current stimulation (tDCS) decreases FC compared with baseline. a Decreased connectivity between the left VPL (seed in white) and left S1, left parahippocampal gyrus, and amygdala after sham tDCS compared with baseline. Plots show changes in FC from baseline to after the sham treatment period for each patient with fibromyalgia. b Decreased connectivity between the right VPL (seed in white) and left IPL. c Decreased connectivity between the PAG (seed in white) and precuneus. Data shown are Fisher-transformed $r$ values. VPL ventral posterolateral, S1 primary somatosensory cortex, parahipp parahippocampal gyrus, amyg amygdala, IPL inferior parietal lobule, PAG periaqueductal gray, L left, $R$ right, FC functional connectivity

Table 3 Correlations between change in FC and change in clinical pain (VAS) for sham versus baseline

\begin{tabular}{|c|c|c|c|c|c|c|c|}
\hline $\begin{array}{l}\text { Seed } \\
\text { FC region }\end{array}$ & \multicolumn{3}{|c|}{$\begin{array}{l}\text { MNI coordinates } \\
(x, y, z)\end{array}$} & \multirow[t]{2}{*}{$r$ Value } & \multirow[t]{2}{*}{$T$} & \multirow[t]{2}{*}{$\begin{array}{l}\text { Cluster } \\
\text { size }\end{array}$} & \multirow[t]{2}{*}{$\begin{array}{l}\text { Cluster } \\
p \text { value }\end{array}$} \\
\hline \multicolumn{4}{|l|}{ L VL thalamus } & & & & \\
\hline $\begin{array}{l}\text { L posterior } \\
\text { insula }\end{array}$ & -48 & -12 & 0 & 0.979 & 12.33 & 313 & 0.001 FWE \\
\hline \multicolumn{8}{|l|}{ R VPL thalamus } \\
\hline R M1 & 56 & -12 & 42 & 0.969 & 9.85 & 603 & 0.000 FWE \\
\hline R S1 & 44 & -34 & 54 & 0.917 & 7.46 & 235 & 0.008 FWE \\
\hline L M1 & -46 & -6 & 26 & 0.936 & 9.41 & 158 & 0.046 FWE \\
\hline \multicolumn{8}{|c|}{ R S1 (postcentral gyrus) } \\
\hline Cerebellum & 36 & -52 & -20 & 0.937 & 7.63 & 355 & 0.001 FWE \\
\hline
\end{tabular}

FC functional connectivity, VAS visual analog scale, $L$ left, $R$ right, $V L$ ventral lateral, VPL ventral posterolateral, M1 primary motor cortex, S1 primary somatosensory cortex, MNI Montreal Neurological Institute $T$ test statistic
FWE) (Additional file 1: Figure S4 and Table S4). There were no significant increases in FC.

We did not find any regions that met whole-brain correction in a regression analysis measuring changes in connectivity in relation to changes in pain after real tDCS compared with sham. However, there were regions that met significance using SVC with a priori ROIs (Table 5). The change in connectivity between the left VPL thalamus and left M1/S1 ( $p=0.007$ FWE, SVC) and right posterior insula ( $p=0.007 \mathrm{FWE}, \mathrm{SVC})$ was positively correlated with the change in clinical pain (Fig. 5). The change in left VL thalamus to right posterior insula connectivity was also positively correlated with change in pain $(p=0.022$ FWE, SVC). Patients with reduced connectivity between the VL/VPL thalamus and M1/S1 and posterior insula had a greater reduction in pain intensity after real tDCS. In post hoc analyses, these changes in connectivity were also correlated with the change in McGill clinical pain (Additional file 1: Table S6). However, there were no significant relationships between FC and changes in positive or negative affect. In an 

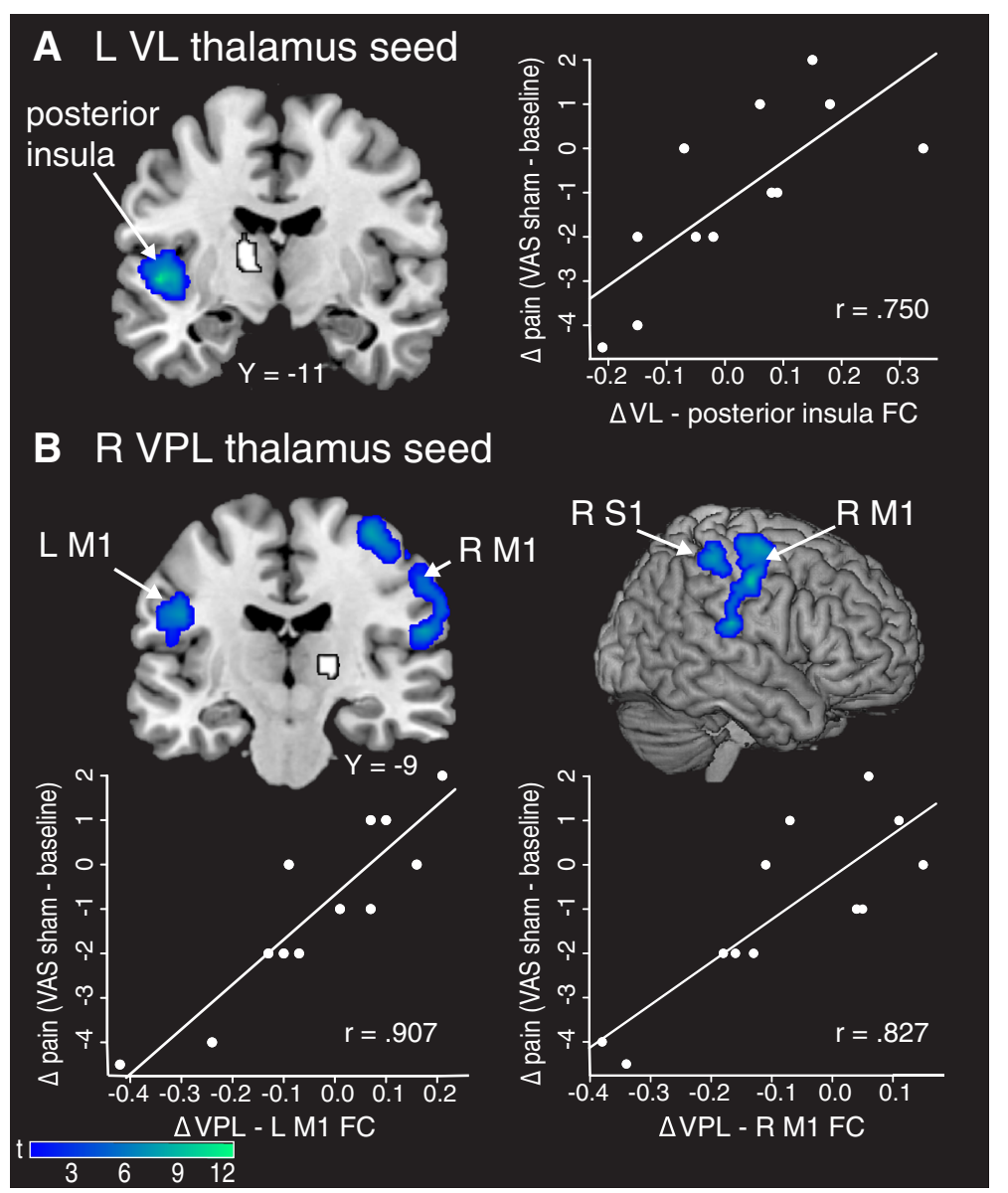

Fig. $\mathbf{3}$ Correlations between changes in FC and changes in clinical pain after sham transcranial direct current stimulation (tDCS). a Decreased FC between the left VL thalamus (seed in white) and left posterior insula was correlated with a reduction in clinical pain after sham tDCS. b Decreased FC between the right VPL thalamus (seed in white) and left M1, right M1, and right S1 correlated with reduced clinical pain after sham tDCS. Data shown are Fisher-transformed $r$ values. VL ventral lateral, VPL ventral posterolateral, M1 primary motor cortex, S1 primary somatosensory cortex, VAS visual analogue scale, $L$ left, $R$ right, $F C$ functional connectivity

Table 4 Main effect of real tDCS on FC

\begin{tabular}{|c|c|c|c|c|c|c|}
\hline \multirow{2}{*}{$\begin{array}{l}\text { Seed } \\
\text { FC region } \\
\text { Sham }>\text { real }\end{array}$} & \multicolumn{3}{|c|}{$\begin{array}{l}\text { MNI coordinates } \\
(x, y, z)\end{array}$} & \multirow[t]{2}{*}{$T$} & \multirow[t]{2}{*}{$\begin{array}{l}\text { Cluster } \\
\text { size }\end{array}$} & \multirow[t]{2}{*}{$\begin{array}{l}\text { Cluster } \\
p \text { value }\end{array}$} \\
\hline & & & & & & \\
\hline \multicolumn{7}{|l|}{ L VL thalamus } \\
\hline mPFC & 4 & 56 & 8 & 5.66 & 362 & 0.006 FWE \\
\hline L SMA & -2 & 24 & 56 & 5.49 & 228 & 0.043 FWE \\
\hline L OFG & -10 & 40 & -22 & 5.91 & 185 & $0.08 \mathrm{FWE}^{\mathrm{a}}$ \\
\hline \multicolumn{7}{|l|}{$\mathrm{R} V \mathrm{~L}$ thalamus } \\
\hline Cerebellum & 16 & -46 & -22 & 6.88 & 1122 & 0.000 FWE \\
\hline L SMA & -6 & 22 & 58 & 6.16 & 313 & 0.016 FWE \\
\hline \multicolumn{7}{|l|}{ Sham $<$ real } \\
\hline N.S. & & & & & & \\
\hline
\end{tabular}

$t D C S$ transcranial direct current stimulation, $F C$ functional connectivity, $L$ left, $R$ right, $V L$ ventral lateral, $M P F C$ medial prefrontal cortex, SMA supplementary motor area, OFG orbitofrontal gyrus, N.S. not significant, MNI Montreal Neurological Institute $T$ test statistic

${ }^{a}$ Trend at $p<0.05$ familywise error correction for multiple comparisons analysis in which we examined changes in connectivity and changes in clinical pain from baseline to real tDCS, we found that patients with reduced connectivity between left S1 and left SMA had a greater reduction in clinical pain ( $p=0.013$ FWE) (Additional file 1: Figure S5 and Table S5). Again, there were no significant relationships between increases in connectivity and decreases in clinical pain, neither between sham and real tDCS nor between baseline and real tDCS (Additional file 1: Figure S6).

\section{Discussion}

This study shows, for the first time to our knowledge, that a clinically relevant schedule of repetitive M1 tDCS sessions alters FC in patients with FM. Real tDCS (versus sham) reduced FC between the VL thalamus and SMA, mPFC, and the cerebellum. These changes in FC were distinct from those observed after sham tDCS. Sham tDCS (compared with baseline) decreased connectivity between the VPL thalamus and S1, IPL, and the 


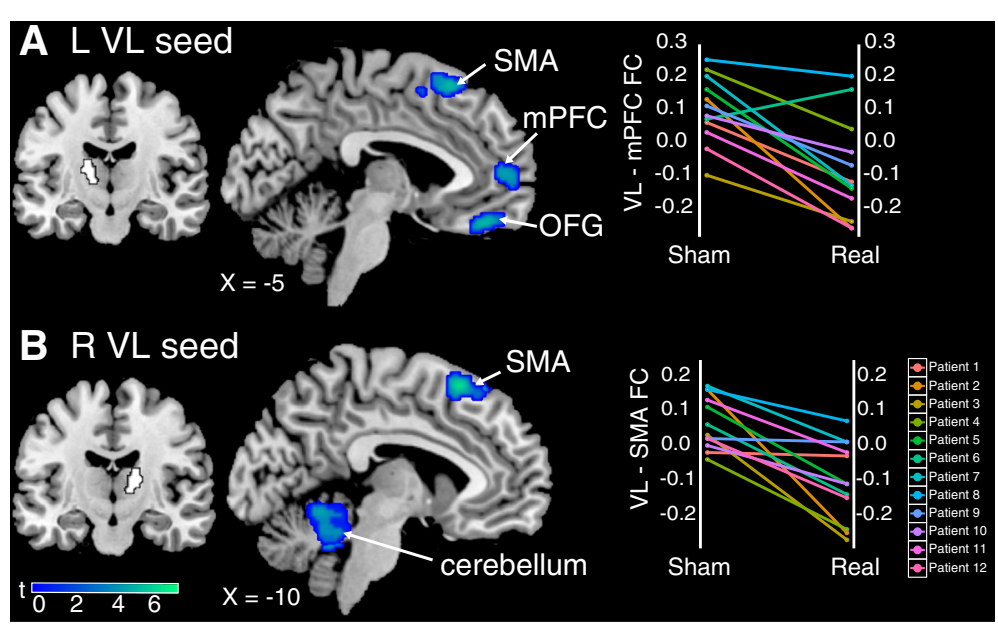

Fig. 4 Real transcranial direct current stimulation (tDCS) decreases FC compared with sham. a Decreased connectivity between the left VL (seed in white) and SMA and MPFC after real tDCS. b Decreased connectivity between the right VL (seed in white) and SMA and cerebellum after real tDCS. Plots show changes in FC between sham and real tDCS for each patient with fibromyalgia. Data shown are Fisher-transformed $r$ values. OFG orbitofrontal gyrus, $V L$ ventral lateral, SMA supplementary motor area, MPFC medial prefrontal cortex, $L$ left, $R$ right, $F C$ functional connectivity

parahippocampal gyrus/amygdala and between the PAG and precuneus. However, after both sham and active tDCS, we found a relationship between decreases in FC among pronociceptive brain regions and reductions in clinical pain. Patients with decreased connectivity between the VL thalamus and posterior insula and between the VPL thalamus and M1/S1 had greater reductions in clinical pain after sham and real tDCS. In addition, our data indicate that patients with FM with stronger baseline connectivity between left M1 and left VL thalamus, between left $\mathrm{S} 1$ and left anterior insula, and between left VL thalamus and PAG had a better analgesic response across the entire study. Although we saw distinct main effects for sham and active tDCS, the overlapping results related to clinical changes in pain may point to a shared placebo response in both sham and active conditions. A summary of the results is depicted in Fig. 6.

Table 5 Correlations between change in FC and change in clinical pain (VAS) for real versus sham

\begin{tabular}{llllllll}
\hline $\begin{array}{l}\text { Seed } \\
\text { FC region }\end{array}$ & $\begin{array}{l}\text { MNI coordinates } \\
(x, y, z)\end{array}$ & $r$ Value & $T$ & $\begin{array}{l}\text { Cluster } \\
\text { size }\end{array}$ & $\begin{array}{l}\text { Cluster } \\
p \text { value }\end{array}$ \\
\hline $\begin{array}{l}\text { L VL thalamus } \\
\begin{array}{l}\text { R posterior } \\
\text { insula }\end{array}\end{array}$ & 42 & -24 & 20 & 0.887 & 5.08 & 5 & $0.022 \mathrm{FWE}^{\mathrm{a}}$ \\
$\begin{array}{l}\text { L VPL thalamus } \\
\text { L M1/S1 }\end{array}$ & -48 & -18 & 50 & 0.923 & 6.45 & 89 & $0.007 \mathrm{FWE}^{\mathrm{a}}$ \\
$\begin{array}{l}\mathrm{R} \text { posterior } \\
\text { insula }\end{array}$ & 46 & -30 & 24 & 0.927 & 7.64 & 23 & $0.007 \mathrm{FWE}^{\mathrm{a}}$ \\
\end{tabular}

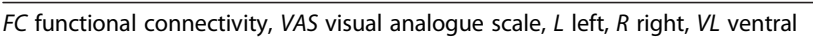
lateral, VPL ventral posterolateral, $M 1$ primary motor cortex, S1 primary somatosensory cortex, MNI Montreal Neurological Institute $T$ test statistic

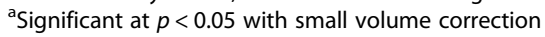

Our findings are somewhat at odds with the existing literature. For example, researchers in some studies have reported increases in thalamic blood flow [32] or increased M1-thalamus connectivity after M1 stimulation $[17,33]$. We suggest that these conflicting results can be explained by the timing of stimulation and measurement. In the other studies, neural activity was measured during or immediately after M1 stimulation, which likely has a different neural signature than after 1 week of repetitive stimulation. In support of this notion, GarcíaLarrea and colleagues noted that thalamic blood flow reverted to baseline levels 30 minutes after M1 stimulation stopped [32]. Therefore, the initial or acute changes in thalamic activity may cause a cascade of other events that are important for analgesia [34], leading to the distinct long-term changes that we observed.

How might M1 stimulation promote analgesia in patients with chronic pain? One hypothesis states that M1 stimulation suppresses pain perception directly by inhibiting activity in the lateral thalamus [35, 36]. Compared with healthy control subjects, patients with FM have increased activity in pain-processing structures during experimental pain and increased connectivity in ascending pronociceptive pathways at rest (see [37] for a recent review on neuroimaging findings in FM), although the specific role of the thalamus in FM remains unclear. Both increases and decreases in thalamic activity or connectivity during experimental pain or at rest have been reported $[3,4,38,39]$. In the present study, we found that strong M1-VL thalamus connectivity at baseline predicted a greater reduction in pain across sham and real tDCS periods. This is consistent with work in central poststroke pain, where analgesic response to 


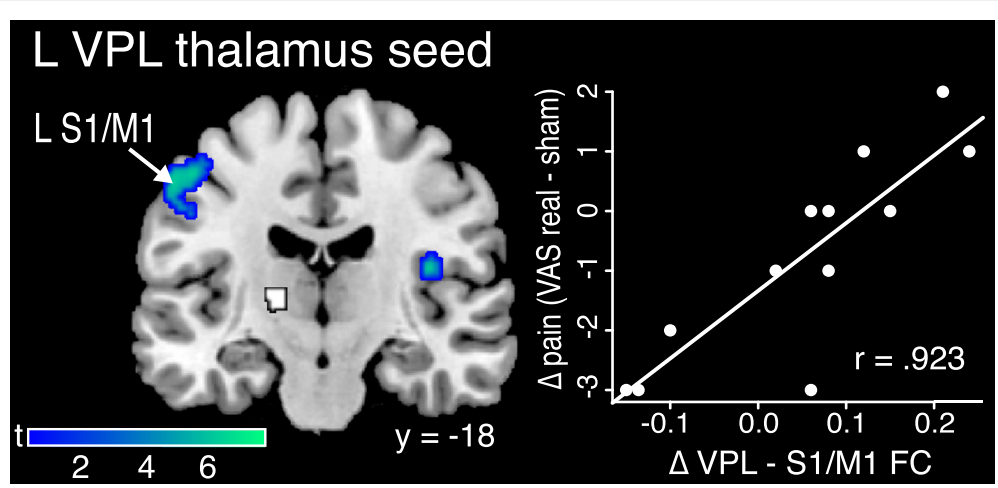

Fig. 5 Correlation between change in FC and change in clinical pain after real transcranial direct current stimulation (tDCS). Patients with reduced FC between the L VPL thalamus (seed in white) and left S1/M1 and right posterior insula had greater reductions in clinical pain after real tDCS compared with sham (displayed at $p=0.005$ ). Data shown are Fisher-transformed $r$ values. VPL ventral posterolateral, S1 primary somatosensory cortex, M1 primary motor cortex, VAS visual analogue scale, $L$ left, $R$ right, FC functional connectivity

repetitive transcranial magnetic stimulation over M1 was found to be best in patients with intact thalamocortical tracts (as measured by diffusion tensor imaging) [22, 23]. Invasive MCS decreases thalamic hyperactivity [40], likely by activating GABAergic divisions of the thalamus and increasing inhibition [41]. In healthy rodents and in a rodent model of neuropathic pain, MCS decreased the firing rate of VPL thalamic neurons specifically $[42,43]$. In a previous study of the same patients reported here, we found a trend toward decreased Glx in the bilateral thalamus after real tDCS compared with sham [24]. The decreases in FC between the thalamus and SMA, MPFC, and cerebellum after real tDCS in this study lends support to the hypothesis that M1 stimulation disrupts thalamic activity.

Another hypothesis is that M1 stimulation causes analgesia indirectly via the facilitation of descending antinociceptive pathways and release of endogenous opioids [34]. Maarrawi and colleagues hypothesized that patients with neuropathic pain who have higher levels of endogenous opioids at baseline would be least likely to benefit from any additional opioid release caused by MCS, and, indeed, patients with lower baseline binding potential for an opioid agonist (reflecting either fewer opioid receptors or higher levels of endogenous opioids) in the thalamus, insula, and PAG were the least likely to benefit from MCS [20]. The relationship between opioids and BOLD fMRI signaling deserves further study, but in healthy controls morphine administration decreases activity in S1, thalamus, and PAG [44] while naloxone (an opioid antagonist) increases activity in S1, thalamus, insula, and PAG [45]. In our study, stronger M1-thalamus, S1-insula, and thalamus-PAG connectivity at baseline predicted a better treatment response. Because both sham and real tDCS also cause endogenous opioid release [14], this finding may suggest that patients with connectivity between regions under the stimulating anode (M1/S1) and regions with a high density of opioid receptors are the most likely to benefit from tDCS. We also found decreases in FC in many of these regions after sham and real tDCS, which could also reflect opioid release.

Placebo analgesia is a well-documented psychobiological event, and imaging studies have revealed overlap between brain networks involved in pain processing and those implicated in the placebo response [46, 47]. The decreases in FC found after sham (compared with baseline) are consistent with previous studies of placebo analgesia showing decreased activity in the thalamus, S1, amygdala, and insula [48-50]. Importantly, the similarity of FC results related to changes in clinical pain between baseline and sham and between sham and real sessions may suggest a shared placebo component across conditions. However, the changes in FC after the sham period in this study cannot be interpreted solely as a placebo effect, because we lacked a control group that received no treatment for comparison. Therefore, any changes from baseline to sham could also reflect regression to the mean or the natural course of the disease [51]. Regression to the mean also may account for a portion of the change in clinical pain after both the sham and real tDCS periods.

Our study was limited by the small sample size, which may have contributed to the lack of a significant clinical effect between sham and active conditions and reduced statistical power. In this pilot study, we did not aim to validate the efficacy of M1 tDCS as a treatment for FM; rather, our goal was to determine if a clinically relevant schedule of tDCS sessions altered resting state FC in patients with FM. However, as with most pain treatments wherein only a portion have a clinically meaningful response, half of the patients in the present study did report a drop in pain on the VAS (active-sham), and this change was correlated with reductions in thalamocortical connectivity. This study is significantly limited 


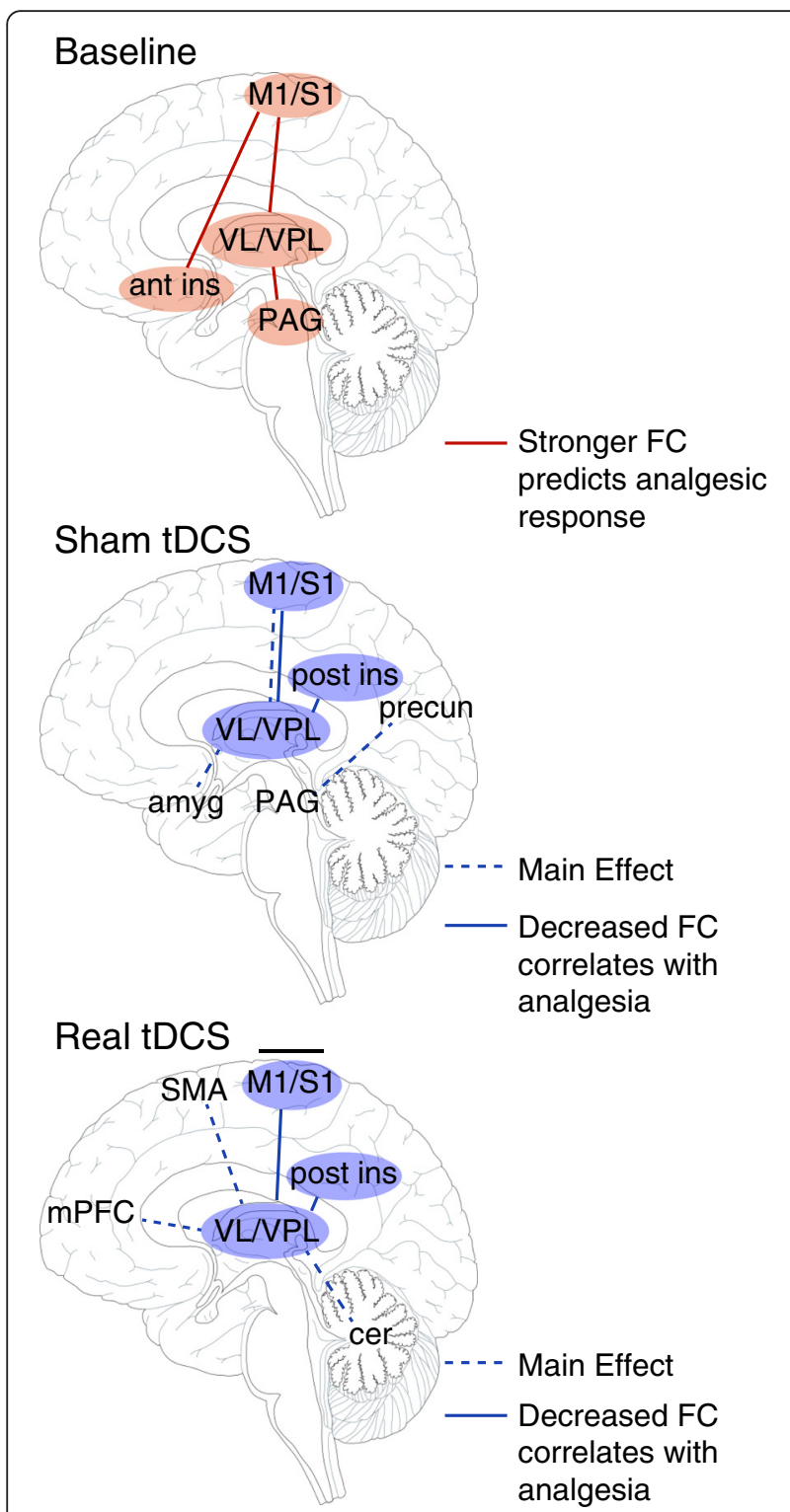

Fig. 6 Summary of results. Stronger corticothalamic FC and FC between regions with high densities of opioid receptors at baseline predicted a better clinical response across the entire study. Changes in FC were observed after sham transcranial direct current stimulation (tDCS), which could be attributed to placebo analgesia or regression to the mean. Real tDCS caused some distinct long-lasting changes in FC (compared with sham) and may have relieved pain via the inhibition of thalamic activity and subsequent decreases in FC, both of which could have been caused by the release of endogenous opioids. M1 primary motor cortex, S1 primary somatosensory cortex, VL ventral lateral, VPL ventral posterolateral, Ant Ins anterior insula, PAG periaqueductal gray, Post Ins posterior insula, Amyg amygdala, Precun precuneus, SMA supplemental motor area, MPFC medial prefrontal cortex, Cer cerebellum, FC functional connectivity

by the lack of counterbalancing between sham and active conditions. Therefore, we cannot rule out carryover effects from the sham period. However, sham and active phases were separated by a washout period of at least
1 week, so we find this unlikely. Another limitation is that the stimulation sessions were single-blinded. Finally, research has shown that stimulation for 30 seconds at the beginning and end of the sham condition can mimic the sensation during active treatment and that patients cannot differentiate between sham and real stimulation at $1 \mathrm{~mA}$ [52]. However, the credibility of sham tDCS and the effectiveness of patient blinding at $2 \mathrm{~mA}$ has recently been questioned [11]. Given our repeated-measures design, it is likely that the patients became aware of the condition differences. Our results should be interpreted with caution, and additional studies designed to replicate our findings are needed.

\section{Conclusions}

Our results support the hypothesis that repetitive M1 tDCS causes lasting changes in FC and may relieve pain by altering thalamic activity. Analgesia may result from the inhibition of thalamic activity and subsequent decreases in FC, both of which could be caused by the release of endogenous opioids. Future studies that combine fMRI and positron emission tomography within the same patients after repetitive M1 tDCS are needed to test this hypothesis. It is possible that there is a significant placebo component common to both sham and real tDCS. Future studies should include a no-treatment control group to test this hypothesis. It remains to be seen if similar changes in FC are observed for tDCS in other chronic pain conditions.

\section{Additional file}

Additional file 1: (DOCX $3033 \mathrm{~kb})$

\section{Abbreviations}

ANOVA: analysis of variance; BOLD: blood oxygen level-dependent; Cl: confidence interval; FC: functional connectivity; FM: fibromyalgia; fMRI: functional magnetic resonance imaging; FWE: familywise error; Glx: glutamate + glutamine; IPL: inferior parietal lobule; M1: primary motor cortex; MCS: motor cortex stimulation; MNI: Montreal Neurological Institute; MPFC: medial prefrontal cortex; N.S.: not significant; OFG: orbitofrontal gyrus; PAG: periaqueductal gray; PANAS: Positive and Negative Affect Schedule; ROI: region of interest; S1: primary somatosensory cortex; SE: standard error; SMA: supplementary motor area; SVC: small volume correction; tDCS: transcranial direct current stimulation; TR: repetition time; TE: echo time; VAS: visual analogue scale; VL: ventral lateral; VPL: ventral posterolateral.

\section{Competing interests}

REH has consulted for and received grant funding from Pfizer Inc. DJC has consulted for Pfizer Inc., Cerephex Corporation and Eli Lilly and Company.

\section{Authors' contributions}

DJC, JKZ, REH, and AFD designed the research. TDN, BRF, and AFD performed the research. CMC analyzed the data. All authors contributed to writing the manuscript. All authors read and approved the final manuscript.

\section{Acknowledgments}

This study was funded by a Michigan Institute for Clinical \& Health Research Clinical Trial Planning Program grant and a Clinical and Translational Science Awards high-tech funding grant, University of Michigan (Principal Investigators 
AFD and REH). CMC was supported by grant T32 NS076401. REH was supported by National Institutes of Health (NIH)/National Center for Complementary and Alternative Medicine grant R01 AT007550. AFD was supported by NIH/National Institute of Dental and Craniofacial Research grant R56 DE022637-01. The authors thank Suzan Lowe, B.A., R.T., for expert technical assistance with fMR data collection.

\section{Author details}

'Neuroscience Graduate Program, University of Michigan, Ann Arbor, Ml, USA. ${ }^{2}$ Chronic Pain and Fatigue Research Center, Department of Anesthesiology, University of Michigan, Ann Arbor, MI, USA. ${ }^{3}$ Headache \& Orofacial Pain Effort (H.O.P.E.) Lab, Department of Biologic and Materials Sciences, School of Dentistry, University of Michigan, Ann Arbor, MI, USA. ${ }^{4}$ Michigan Center for Oral Health Research, Michigan Institute for Clinical \& Health Research, University of Michigan, Ann Arbor, MI, USA. ${ }^{5}$ Ann Arbor VA Healthcare System, Ann Arbor, MI, USA. ${ }^{6}$ Russell H. Morgan Department of Radiology, Johns Hopkins University, Baltimore, MD, USA. 'Department of Radiology, University of Michigan, Ann Arbor, MI, USA. ${ }^{8}$ Division of Rheumatology, Department of Internal Medicine, University of Michigan, Ann Arbor, MI, USA. ${ }^{9}$ University Neuropsychiatric Institute, Department of Psychiatry, University of Utah Health Sciences Center, Salt Lake City, UT, USA.

\section{Received: 5 October 2015 Accepted: 18 January 2016}

\section{Published online: 03 February 2016}

\section{References}

1. Wolfe F, Smythe HA, Yunus MB, Bennett RM, Bombardier C, Goldenberg DL, et al. The American College of Rheumatology 1990 criteria for the classification of fibromyalgia. Arthritis Rheum. 1990;33:160-72.

2. Schmidt-Wilcke T, Clauw DJ. Fibromyalgia: from pathophysiology to therapy. Nat Rev Rheumatol. 2011;7:518-27.

3. Jensen KB, Kosek E, Petzke F, Carville S, Fransson P, Marcus H, et al. Evidence of dysfunctional pain inhibition in fibromyalgia reflected in rACC during provoked pain. Pain. 2009;144:95-100.

4. Jensen KB, Loitoile R, Kosek E, Petzke F, Carville S, Fransson P, et al. Patients with fibromyalgia display less functional connectivity in the brain's pain inhibitory network. Mol Pain. 2012;8:32.

5. Napadow V, Kim J, Clauw DJ, Harris RE. Decreased intrinsic brain connectivity is associated with reduced clinical pain in fibromyalgia. Arthritis Rheum. 2012;64:2398-403.

6. Napadow V, LaCount L, Park K, As-Sanie S, Clauw DJ, Harris RE. Intrinsic brain connectivity in fibromyalgia is associated with chronic pain intensity. Arthritis Rheum. 2010;62:2545-55.

7. Castillo Saavedra L, Mendonca M, Fregni F. Role of the primary motor cortex in the maintenance and treatment of pain in fibromyalgia. Med Hypotheses. 2014;83:332-6.

8. Fagerlund AJ, Hansen OA, Aslaksen PM. Transcranial direct current stimulation as a treatment for patients with fibromyalgia. Pain. 2015;156:62-71.

9. Fregni F, Gimenes R, Valle AC, Ferreira MUL, Rocha RR, Natalle L, et al. A randomized, sham-controlled, proof of principle study of transcranial direct current stimulation for the treatment of pain in fibromyalgia. Arthritis Rheum. 2006:54:3988-98.

10. Valle A, Roizenblatt S, Botte S, Zaghi S, Riberto M, Tufik S, et al. Efficacy of anodal transcranial direct current stimulation (tDCS) for the treatment of fibromyalgia: results of a randomized, sham-controlled longitudinal clinical trial. J Pain Manag. 2009;2:353-61.

11. O'Connell NE, Wand BM, Marston L, Spencer S, Desouza LH. Non-invasive brain stimulation techniques for chronic pain. Cochrane Database Syst Rev. 2014:4:CD008208.

12. Petrovic $P$, Kalso E, Petersson KM, Ingvar M. Placebo and opioid analgesia_imaging a shared neuronal network. Science. 2002;295:1737-40.

13. Wager TD, Scott DJ, Zubieta JK. Placebo effects on human $\mu$-opioid activity during pain. Proc Natl Acad Sci U S A. 2007;104:11056-61.

14. DosSantos MF, Martikainen IK, Nascimento TD, Love TM, DeBoer MD, Schambra HM, et al. Building up analgesia in humans via the endogenous $\mu$-opioid system by combining placebo and active tDCS: a preliminary report. PLoS One. 2014;9:e102350.

15. Dayan E, Censor N, Buch ER, Sandrini M, Cohen LG. Noninvasive brain stimulation: from physiology to network dynamics and back. Nat Neurosci. 2013;16:838-44.
16. Polanía R, Paulus W, Nitsche MA. Reorganizing the intrinsic functional architecture of the human primary motor cortex during rest with non-invasive cortical stimulation. PLoS One. 2012;7:e30971.

17. Polanía R, Paulus W, Nitsche MA. Modulating cortico-striatal and thalamocortical functional connectivity with transcranial direct current stimulation. Hum Brain Mapp. 2012;33:2499-508.

18. Lindenberg R, Nachtigall L, Meinzer M, Sieg MM, Flöel A. Differential effects of dual and unihemispheric motor cortex stimulation in older adults. J Neurosci. 2013;33:9176-83.

19. Sehm B, Schäfer A, Kipping J, Margulies D, Conde V, Taubert M, et al. Dynamic modulation of intrinsic functional connectivity by transcranial direct current stimulation. J Neurophysiol. 2012;108:3253-63.

20. Maarrawi J, Peyron R, Mertens P, Costes N, Magnin M, Sindou M, et al. Brain opioid receptor density predicts motor cortex stimulation efficacy for chronic pain. Pain. 2013;154:2563-8.

21. Maarrawi J, Peyron R, Mertens P, Costes N, Magnin M, Sindou M, et al. Motor cortex stimulation for pain control induces changes in the endogenous opioid system. Neurology. 2007;69:827-34.

22. Goto T, Saitoh $Y$, Hashimoto N, Hirata M, Kishima H, Oshino S, et al. Diffusion tensor fiber tracking in patients with central post-stroke pain; correlation with efficacy of repetitive transcranial magnetic stimulation. Pain. 2008:140:509-18.

23. Ohn SH, Chang WH, Park CH, Kim ST, Lee Jl, Pascual-Leone A, et al. Neural correlates of the antinociceptive effects of repetitive transcranial magnetic stimulation on central pain after stroke. Neurorehabil Neural Repair. 2012;26:344-52

24. Foerster BR, Nascimento TD, DeBoer M, Bender MA, Rice IC, Truong DQ, et al. Excitatory and inhibitory brain metabolites as targets and predictors of effective motor cortex tDCS therapy in fibromyalgia. Arthritis Rheumatol. 2015;67:576-81.

25. Zhang D, Snyder AZ, Shimony JS, Fox MD, Raichle ME. Noninvasive functional and structural connectivity mapping of the human thalamocortical system. Cereb Cortex. 2010;20:1187-94.

26. Brunoni AR, Fregni F. Clinical trial design in non-invasive brain stimulation psychiatric research. Int J Methods Psychiatr Res. 2011;20:e19-30.

27. Melzack R. The short-form McGill pain questionnaire. Pain. 1987;30:191-7.

28. Watson D, Clark LA, Tellegen A. Development and validation of brief measures of positive and negative affect: the PANAS scales. J Pers Soc Psychol. 1988:54:1063-70.

29. DaSilva AF, Volz MS, Bikson M, Fregni F. Electrode positioning and montage in transcranial direct current stimulation. J Vis Exp. 2011;51:2744. doi:10.3791/2744

30. Whitfield-Gabrieli S, Nieto-Castanon A. Conn: a functional connectivity toolbox for correlated and anticorrelated brain networks. Brain Connect. 2012:2·125-41.

31. Vaseghi B, Zoghi M, Jaberzadeh S. Does anodal transcranial direct current stimulation modulate sensory perception and pain? A meta-analysis study. Clin Neurophysiol. 2014;125:1847-58.

32. García-Larrea L, Peyron R, Mertens P, Gregoire MC, Lavenne F, Le Bars D, et al. Electrical stimulation of motor cortex for pain control: a combined PETscan and electrophysiological study. Pain. 1999:83:259-73.

33. Sours C, Alon G, Roys S, Gullapalli RP. Modulation of resting state functional connectivity of the motor network by transcranial pulsed current stimulation. Brain Connect. 2014;4:157-65.

34. Garcia-Larrea L, Peyron R. Motor cortex stimulation for neuropathic pain: From phenomenology to mechanisms. Neuroimage. 2007;37 Suppl 1:S71-9.

35. Kuo MF, Paulus W, Nitsche MA. Therapeutic effects of non-invasive brain stimulation with direct currents (tDCS) in neuropsychiatric diseases. Neuroimage. 2014;85:948-60.

36. Plow EB, Pascual-Leone A, Machado A. Brain stimulation in the treatment of chronic neuropathic and non-cancerous pain. J Pain. 2012;13:411-24.

37. Cagnie B, Coppieters I, Denecker S, Six J, Danneels L, Meeus M. Central sensitization in fibromyalgia? A systematic review on structural and functional brain MRI. Semin Arthritis Rheum. 2014:44:68-75.

38. Burgmer M, Pogatzkizahn E, Gaubitz M, Wessoleck E, Heuft G, Pfleiderer B. Altered brain activity during pain processing in fibromyalgia. Neuroimage. 2009;44:502-8.

39. Cifre I, Sitges C, Fraiman D, Muñoz MÁ, Balenzuela P, González-Roldán A, et al. Disrupted functional connectivity of the pain network in fibromyalgia. Psychosom Med. 2012:74:55-62.

40. Tsubokawa T, Katayama Y, Yamamoto T, Hirayama T, Koyama S. Chronic motor cortex stimulation in patients with thalamic pain. J Neurosurg. 1993:78:393-401. 
41. Lucas JM, Ji Y, Masri R. Motor cortex stimulation reduces hyperalgesia in an animal model of central pain. Pain. 2011;152:1398-407.

42. Pagano RL, Assis DV, Clara JA, Alves AS, Dale CS, Teixeira MJ, et al. Transdural motor cortex stimulation reverses neuropathic pain in rats: a profile of neuronal activation. Eur J Pain. 2011;15:268. e1-e14.

43. Pagano RL, Fonoff ET, Dale CS, Ballester G, Teixeira MJ, Britto LRG. Motor cortex stimulation inhibits thalamic sensory neurons and enhances activity of PAG neurons: possible pathways for antinociception. Pain. 2012;153:2359-69.

44. Becerra L, Harter K, Gonzalez RG, Borsook D. Functional magnetic resonance imaging measures of the effects of morphine on central nervous system circuitry in opioid-naive healthy volunteers. Anesth Analg. 2006;103:208-16.

45. Borras MC, Becerra L, Ploghaus A, Gostic JM, DaSilva A, Gonzalez RG, et al. fMRI measurement of CNS responses to naloxone infusion and subsequent mild noxious thermal stimuli in healthy volunteers. J Neurophysiol. 2004;91:2723-33.

46. Meissner K, Bingel U, Colloca L, Wager TD, Watson A, Flaten MA. The placebo effect: advances from different methodological approaches. J Neurosci. 2011;31:16117-24.

47. Tracey I. Getting the pain you expect: mechanisms of placebo, nocebo and reappraisal effects in humans. Nat Med. 2010;16:1277-83.

48. Price DD, Craggs J, Nicholas Verne G, Perlstein WM, Robinson ME. Placebo analgesia is accompanied by large reductions in pain-related brain activity in irritable bowel syndrome patients. Pain. 2007;127:63-72.

49. Wager $T D$, Atlas $L Y$, Leotti $L A$, Rilling JK. Predicting individual differences in placebo analgesia: contributions of brain activity during anticipation and pain experience. J Neurosci. 2011;31:439-52.

50. Wager TD, Rilling JK, Smith EE, Sokolik A, Casey KL, Davidson RJ, et al. Placebo-induced changes in fMRI in the anticipation and experience of pain. Science. 2004;303:1162-7.

51. Wager TD, Fields HL. Placebo analgesia. In: McMahon SB, Koltzenburg M, Tracey I, Turk D, editors. Wall and Melzack's textbook of pain. 6th ed. Philadelphia: Saunders; 2013. p. 362-74.

52. Gandiga PC, Hummel FC, Cohen LG. Transcranial DC stimulation (tDCS): a tool for double-blind sham-controlled clinical studies in brain stimulation. Clin Neurophysiol. 2006;117:845-50.

\section{Submit your next manuscript to BioMed Central and we will help you at every step:}

- We accept pre-submission inquiries

- Our selector tool helps you to find the most relevant journal

- We provide round the clock customer support

- Convenient online submission

- Thorough peer review

- Inclusion in PubMed and all major indexing services

- Maximum visibility for your research

Submit your manuscript at www.biomedcentral.com/submit

C Biomed Central 